\title{
Hospital readmissions in people with chronic spinal cord injury
}

\author{
G Savic*,1, DJ Short $^{2}$, D Weitzenkamp ${ }^{3}$, S Charlifue $^{3}$ and BP Gardner ${ }^{1}$ \\ ${ }^{1}$ National Spinal Injuries Centre, Stoke Mandeville Hospital, Aylesbury, Buckinghamshire, UK; ${ }^{2}$ Midlands Centre for \\ Spinal Injuries, Robert Jones \& Agnes Hunt Orthopaedic \& District Hospital, Gobowen, Oswestry, Shropshire, UK; \\ ${ }^{3}$ Craig Hospital, Englewood, Colorado, USA
}

\begin{abstract}
Study design: Longitudinal observational.
Objectives: To examine frequency and duration of hospital readmissions in a population based sample of people with chronic spinal cord injury (SCI) and to look at medical reasons necessitating readmissions and factors influencing them.

Setting: National Spinal Injuries Centre, Stoke Mandeville Hospital, Aylesbury, UK and Regional Spinal Injuries Centre, District General Hospital, Southport, UK.

Methods: One-hundred-and-ninety-eight SCI patients, all injured more than 20 years ago, were interviewed on three occasions and their medical records reviewed for the period 19901996.

Results: Between 1990 and 1996, 127 patients (64\% of the sample) required hospital treatment for late medical complications, with 481 readmissions between them and the mean length of stay of 12.03 days per readmission. Only $58 \%$ of all readmissions were into specialised spinal injuries centres. Averaged over the entire sample, the readmission rate was 0.4 readmissions per person at risk per year, and the occupancy rate was 4.9 bed-days per person at risk per year. The most frequent reason for readmissions were urinary tract complications (40.5\% of all readmissions) and the highest bed occupancy was for skin problems (32.2\% of all bed-days). When compared with the non-hospitalised group (36\% of the sample), the readmitted patients had longer duration of paralysis and lower disability and handicap scores as measured by Functional Independence Measure (FIM) and Craig Handicap Assessment \& Reporting Technique (CHART). The subgroups did not differ significantly by neurological grouping or age.

Conclusions: Urinary and skin complications are the two main reasons for hospital readmissions in people with chronic SCI. Risk of readmissions increases with time since injury and with disability and handicap severity. Hospital bed requirements for people with chronic SCI are greater than the amount of clinical provision currently available in specialised spinal centres. In order to meet the needs of the growing SCI population, more specialised spinal injuries care beds will be needed.
\end{abstract}

Spinal Cord (2000) 38, 371-377

Keywords: spinal cord injury; readmission; hospitalisation

\section{Introduction}

Acute spinal cord injury is obviously a devastating life event, what is less obvious is the permanent multisystem impairment which results from the spinal cord damage. This multisystem dysfunction renders the individual susceptible to a range of related complications and requires lifelong management. Effective treatment depends on familiarity with and cumulative experience of spinal cord injury pathophysiology, ie the concentration of expertise constituting a specialised spinal injury unit. Within a fixed number of spinal injury unit beds, priority will almost always be given to accommodating new acute admissions. This means that

*Correspondence: G Savic, National Spinal Injuries Centre, Stoke Mandeville Hospital, Aylesbury, Buckinghamshire HP21 8AL, UK patients with chronic spinal cord injury who require hospital readmissions are either put on waiting lists or, if urgent, admitted to non-specialised units elsewhere.

The purpose of this study was to look at hospital readmissions in patients with chronic SCI and try to determine the amount of clinical provision required.

While a number of papers address this problem in the US, to our knowledge, there are no similar publications about the situation in the UK.

All US studies ${ }^{1-5}$ agree that in the first few years post injury the number of rehospitalisations and the average lengths of stay decrease steadily. Ivie and DeVivo ${ }^{1}$ found that risk of rehospitalisation declined by $13 \%$ in each successive year following initial discharge for years $1-7$ post injury. Samsa et $a l^{2}$ 
reported that the incidence of rehospitalisation decreased rapidly in years $2-5$ post injury and declined less rapidly thereafter, and that occupancy rates followed a similar pattern. The actual number of readmissions and lengths of stay in these studies varied depending on the cohort and the length of time observed. Risk factors and predictors of rehospitalisation (level of injury, age, sex, race, marital status, education, employment, time since injury, ADL, living environment - to name just a few of analysed variables) were also different from one study to the next. Mean time since injury in the mentioned studies was from 1 to 14 years, which is probably one of the reasons for discrepancies in the results.

This study concentrated solely on people injured more than 20 years ago, in order to examine patterns of readmissions in the 'ageing' spinal cord injured population.

\section{Materials and methods}

The study sample consisted of 198 SCI patients who participated in the 1990, 1993 and 1996 Ageing with Spinal Cord Injury Study. ${ }^{6-8}$ The original sample was selected on the basis of the following criteria: All patients had to have been injured more than 20 years ago when first seen for the Ageing Study in 1990, they all sustained a traumatic spinal cord injury, were between 15 and 55 years old at the time of injury, lived in the catchment area of one of the two centres National Spinal Injuries Centre (NSIC), Stoke Mandeville or Regional Spinal Injuries Centre (RSIC), Southport and were admitted to the NSIC or RSIC within 1 year of injury.

Data for this paper were extracted from several sources of information: patients' medical records at their spinal centres, their local general practitioners' medical records and from the patients' interviews for the Ageing Study. Because of the original study design, data for the patients who died between the 1990 and 1996 follow-up were not available for this study.

The data of interest for this paper included patients' sex, age, duration of paralysis, level and completeness of injury, number of readmissions, reasons for readmission and lengths of stay for the period between 1990 and 1996 (when first and last seen for the Ageing Study). Diagnosis and interventions were coded using the International Classification of Disease (1978) ICD-9-CM codes. ${ }^{9}$

Results were presented using descriptive statistics. Readmission and bed occupancy rates were expressed per person at risk per year. Comparison between groups was performed using analysis of variance (ANOVA) and the level of statistical significance was set at $P<0.05$ value.

\section{Results}

Of all the patients who fulfilled the original study criteria and entered the Ageing with Spinal Cord Injury
Study group, 198 patients participated on all three occasions, in 1990, 1993 and 1996. Their results are presented here.

\section{Patient characteristics}

The sample was predominantly $(84.8 \%)$ male. In the 1996 follow-up the mean age was 57.5 years (range $41-81$ ), with $70 \%$ of the patients between the age of 50 and 70 . Table 1 shows patients' age characteristics.

In the 1996 follow-up, all patients had been injured more than 26 years, and $66 \%$ of them more than 30 years. Mean time since injury was 33 years (range $26-$ 52). Years post injury (YPI) grouping is shown in Table 2.

Table 1 Age groups in 1996 follow-up

\begin{tabular}{lcc}
\hline Age group & Number & Per cent \\
\hline$<50$ & 39 & 19.7 \\
$50-59$ & 86 & 43.4 \\
$60-69$ & 53 & 26.8 \\
$>70$ & 20 & 10.1 \\
Total & 198 & 100.0 \\
\hline
\end{tabular}

Table 2 Years post injury (YPI) in 1996 follow-up

\begin{tabular}{lcc}
\hline YPI group & Number & Per cent \\
\hline $26-29$ & 68 & 34.3 \\
$30-39$ & 105 & 53.1 \\
$>40$ & 25 & 12.6 \\
Total & 198 & 100.0 \\
\hline
\end{tabular}

By level and completeness of injury, the patients were divided into three neurological groups ${ }^{10,11}$ (shown in Table 3) as follows:

(1) Those with paraplegia and Frankel/ASIA grade A, B or C;

(2) Those with tetraplegia and Frankel/ASIA grade A, $\mathrm{B}$ or $\mathrm{C}$;

(3) Those with Frankel/ASIA grade D, with either para or tetraplegia.

Table 3 Neurologic groups

\begin{tabular}{lcc}
\hline Neurologic group & Number & Per cent \\
\hline Paraplegic ABC & 97 & 49.0 \\
Tetraplegic ABC & 61 & 30.8 \\
All Ds & 40 & 20.2 \\
Total & 198 & 100.0 \\
\hline
\end{tabular}

In the tetraplegic group there were only four patients with $\mathrm{C} 4$ level of injury, none of whom were ventilator dependent. All the others were below the $\mathrm{C} 4$ level. 
Readmissions during 1990-1996 period

Between 1990 and 1996, 127 patients (64\% of the sample) required one or more hospitalisations for medical complications. Patients' medical records review showed that $58 \%$ of all hospitalisations were in their spinal centres (either NSIC or RSIC), and the other $42 \%$ in different local hospitals.

Between them, the 127 patients had 481 diagnosed conditions requiring hospital treatment. This is a mean of 3.8 readmissions per hospitalised person over the observed 6 year period, with the range from 1 to 15 , and the mode of two readmissions per readmitted person. Averaged over the entire sample, there were 2.43 readmissions per person at risk over the observed 6 year period.

The mean length of stay in hospital was 12.03 days per readmission. The range was very wide, from 1 to 181 days per diagnosis. For the observed 6 year period this amounted to 5786 bed-days for the entire sample, or 45.7 bed-days per hospitalised person. Averaged over the entire sample that would be 28.9 bed-days per person at risk during the 1990-1996 period.

\section{Average annual figures}

For the purpose of estimating future bed requirements, average figures for 1 year were calculated from the results of the 6 year observed period. As numbers of readmissions for each year between 1990 and 1996 fluctuated and did not show a consistent trend in any direction, it was felt safer to derive average yearly figures, rather than to present results of any one particular year for this purpose.

Per year, on the average, there were 80 diagnosed conditions which required hospital treatment in the entire sample, which gave the readmission rate of 0.4 readmissions per person at risk per year. As the mean length of stay was 12.03 days per readmission, total yearly number of bed-days would be 964 for the entire sample, giving the bed occupancy rate of 4.9 bed-days per person at risk in any year between 1990 and 1996.
Reasons for readmissions 1990-1996

The most frequent reasons for readmissions were urinary system complications $(40.5 \%$ of all readmissions), followed by skin problems (17\%), digestive system $(10 \%)$, musculoskeletal system $(8.7 \%)$ and nervous system $(6.9 \%)$ complications.

Readmissions requiring the longest stay in hospital were those for skin problems, with mean length of stay (LOS) of 22.7 days per diagnosis, followed by musculoskeletal system (mean LOS 20.9 days per diagnosis) and nervous system complications (mean LOS 19.8 days per diagnosis).

Frequency of readmissions, bed occupancy and mean lengths of stay by organ systems for the 1990-1996 period are given in Table 4.

Skin problems, even though second in number of readmissions (17\% of all readmissions), had the highest bed occupancy $(32.2 \%$ of all bed-days). Urinary system complication, by far the most frequent reason for readmissions $(40.5 \%$ of all readmissions), accounted for $26.1 \%$ of all bed-days and thus were second in bed occupancy.

The five most frequent reasons for readmission and their bed occupancy are also shown in Figure 1.

\section{Rehospitalised patients}

Results of the 127 patients who required hospital readmissions were further analysed by level and completeness of injury, current age and time since injury, to determine how these patients' characteristics affected frequency of readmissions and lengths of stay.

Table 5 shows frequency of readmissions and lengths of stay analysed by level and completeness of injury. There was no difference in number of readmissions or lengths of stay between paraplegic and tetraplegic patients. Even though patients with very incomplete injuries (Frankel/ASIA grade D) had fewer readmissions than patients with Frankel/ASIA grade $\mathrm{A}, \mathrm{B}$ or $\mathrm{C}$, this difference was not statistically significant. The only significant difference among the readmitted patients was shorter length of stay in hospital in patients with Frankel/ASIA grade D, compared with patients with Frankel/ASIA grade A, B or C $(P=0.005)$.

Table 4 Reasons for readmissions, bed occupancy and lengths of stay (LOS) 1990-1996

\begin{tabular}{lccr}
\hline & Diagnoses $1990-1996$ & Bed-days $1990-1996$ & Mean LOS/diagnosis \\
\hline Urgan system & No $(\%)$ & No $(\%)$ & 7.7 \\
Urinary & $195(40.5)$ & $1511(26.1)$ & 22.7 \\
Digestive & $82(17.0)$ & $1861(32.2)$ & 7.5 \\
Musculoskeletal & $48(10.0)$ & $361(6.2)$ & 20.9 \\
Nervous & $42(8.7)$ & $877(15.2)$ & 19.8 \\
Cardiovascular & $33(6.9)$ & $653(11.3)$ & 7.1 \\
Respiratory & $14(2.9)$ & $100(1.7)$ & 4.1 \\
Endocrine & $11(2.3)$ & $46(0.8)$ & 10.3 \\
Others & $8(1.7)$ & $82(1.4)$ & 6.2 \\
Total & $48(10.0)$ & $295(5.1)$ & 12.03 \\
\hline
\end{tabular}




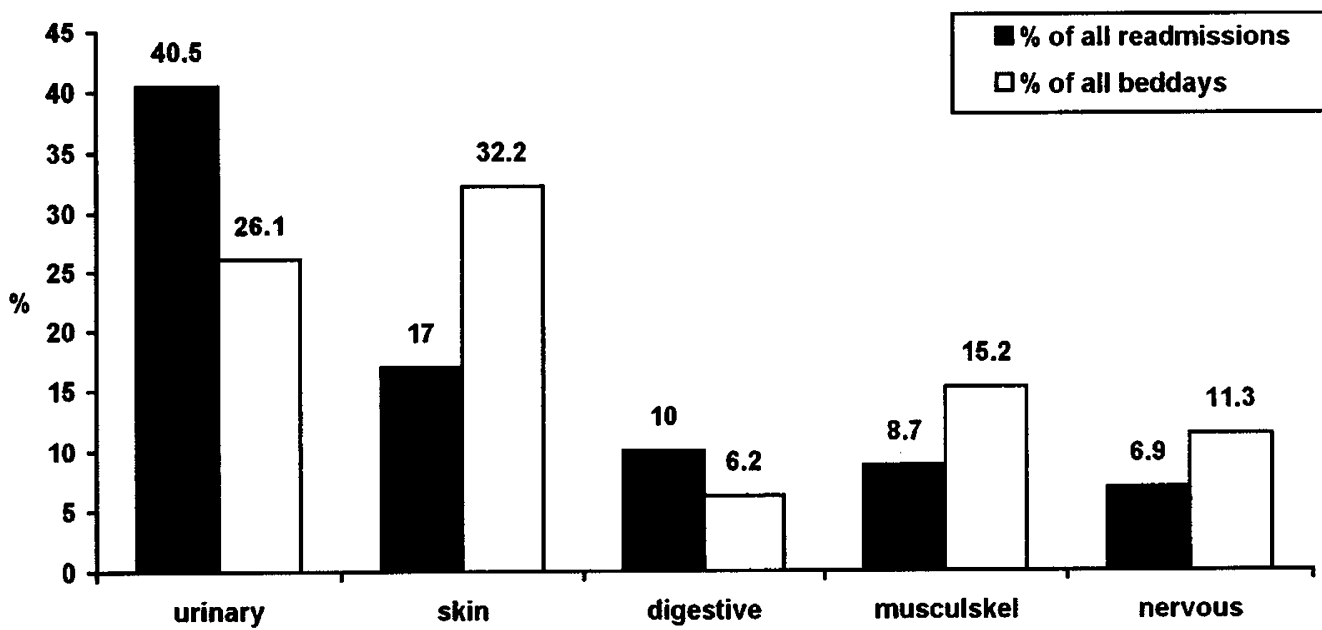

Figure 1 Most frequent reasons for hospital readmission and bed occupancy 1990-1996

Table 5 Number of readmissions and lengths of stay by neurological groups 1990-1996

\begin{tabular}{lcccc}
\hline $\begin{array}{l}\text { Neuro } \\
\text { group }\end{array}$ & $\begin{array}{c}\text { Patients } \\
\text { number }\end{array}$ & $\begin{array}{c}\text { Diagnoses } \\
\text { number }\end{array}$ & $\begin{array}{c}\text { Mean } \\
\text { Diagnoses/ } \\
\text { patient }\end{array}$ & $\begin{array}{c}\text { LOS days/ } \\
\text { diagnosis }\end{array}$ \\
\hline Para ABC & 72 & 263 & 3.65 & 12.5 \\
Tetra ABC & 36 & 163 & 4.53 & 12.65 \\
All Ds & 19 & 55 & 2.89 & 7.18 \\
Total & 127 & 481 & 3.79 & 12.03 \\
$P$-value & & & 0.115 & 0.005 \\
& & & n.s. & \\
\hline
\end{tabular}

n.s., not significant

Tables 6 and 7 show readmissions by age groups and time since injury groups. No statistically significant differences were found among the hospitalised patients in frequency of readmissions and lengths of stay in relation to current age and time since injury.

\section{Rehospitalised: non-hospitalised patients}

The 127 patients who required hospital readmissions were further compared with the 71 patients who were not hospitalised between 1990-1996, to see if they differed when compared by neurological grouping, time since injury, Functional Independence Measure (FIM) score $^{12}$ and Craig Handicap Assessment and Reporting Technique (CHART) score. ${ }^{13}$

There was no statistically significant difference between the hospitalised and non-hospitalised patients when compared by neurological groups $(P=0.061)$.

The groups differed by duration of paralysis $(P=0.012)$, the hospitalised group having been paralysed for 2 years longer than the non-hospitalised group.
Table 6 Number of readmissions and lengths of stay by age groups $1990-1996$

\begin{tabular}{lcccc}
\hline $\begin{array}{l}\text { Age } \\
\text { group }\end{array}$ & $\begin{array}{c}\text { Patients } \\
\text { number }\end{array}$ & $\begin{array}{c}\text { Diagnoses } \\
\text { number }\end{array}$ & $\begin{array}{c}\text { Mean } \\
\text { Diagnoses } \\
\text { patient }\end{array}$ & $\begin{array}{c}\text { LOS days/ } \\
\text { diagnosis }\end{array}$ \\
\hline$<49$ & 36 & 138 & 3.83 & 11.9 \\
$50-59$ & 50 & 177 & 3.56 & 13.1 \\
$60-69$ & 31 & 130 & 4.25 & 11.1 \\
$>70$ & 10 & 36 & 3.36 & 9.5 \\
Total & 127 & 481 & 3.79 & 12.03 \\
$P$-value & & & 0.692 & 0.523 \\
& & & n.s. & n.s. \\
\hline
\end{tabular}

n.s., not significant

Table 7 Number of readmissions and lengths of stay by years post injury (YPI) groups 1990-1996

\begin{tabular}{lcccc}
\hline YPI group & $\begin{array}{c}\text { Patients } \\
\text { number }\end{array}$ & $\begin{array}{c}\text { Diagnoses } \\
\text { number }\end{array}$ & $\begin{array}{c}\text { Average } \\
\text { Diagnoses/ } \\
\text { patient }\end{array}$ & $\begin{array}{c}\text { LOS days/ } \\
\text { diagnosis }\end{array}$ \\
\hline $20-29$ & 68 & 231 & 3.39 & 11.1 \\
$30-39$ & 43 & 191 & 4.45 & 14.0 \\
$>40$ & 16 & 59 & 3.68 & 9.1 \\
Total & 127 & 481 & 3.79 & 12.03 \\
$P$-value & & & 0.203 & 0.262 \\
& & & n.s. & n.s. \\
\hline
\end{tabular}

n.s., not significant

The groups also differed in terms of disability and handicap measures. Total Functional Independent Measure (FIM) score for hospitalised group was 99.43 and for non-hospitalised group 106.18, out of possible $126(P=0.031)$. CHART Physical Independence score was 90.53 for hospitalised and 96.82 for non-hospitalised group, out of possible $100(P=0.003)$ and CHART Occupation score was 60.97 for 
Table 8 Differences between the hospitalised and non-hospitalised patients

\begin{tabular}{|c|c|c|c|c|}
\hline 1996 findings & Hospitalised $\mathrm{n}=127$ & Non-hospitalised $\mathrm{n}=71$ & Total $\mathrm{n}=198$ & $\mathrm{P}$-value \\
\hline Years post injury & 33.8 & 31.8 & 32.94 & 0.012 \\
\hline FIM score & 99.43 & 106.18 & 102.29 & 0.031 \\
\hline CHART physical independence & 90.53 & 96.82 & 93.19 & 0.003 \\
\hline CHART occupation & 60.97 & 75.22 & 66.98 & 0.001 \\
\hline
\end{tabular}

hospitalised and 75.22 for non-hospitalised group, out of possible $100 \quad(P=0.001)$. Table 8 outlines the differences between the groups.

\section{Discussion}

This study examined hospital readmissions in a population based sample of people with chronic spinal cord injury who remained alive over the 6 year followup period. Information on rehospitalisation was well documented in patients' medical records and was cross checked during the medical interview with the patients.

\section{Hospital bed requirements for readmissions}

The primary purpose of this study was to determine the amount of clinical provision required for patients with long-standing spinal cord injury, who may need hospitalisation for late medical complications.

Based on the average annual figures from this study, specialised spinal injuries care bed requirements would be 4.9 bed-days per year for every patient with chronic spinal cord injury, provided all medical complications were treated in spinal centres, or 3 days per person per year if the current interhospital distribution were maintained.

It was not possible to compare our results with those of other authors, as no other publications, to our knowledge, report on readmissions after more than 20 years post injury. Even though some studies have quite a long range of time post injury, they all include readmissions during the first years following injury, which significantly change readmission and occupancy rates.

We compared the readmission and bed occupancy rates of our sample with those in the general population in the UK for 1994/1995. According to the Department of Health Statistics ${ }^{14}$ the hospital admission rate for that year was 0.1655 admissions per person in the general population, compared to 0.4 admissions per person in our sample. This means that a chronic spinal cord injured patient was 2.4 times more likely to be admitted to hospital than a member of the general population in the UK. Once admitted, SCI patients also stayed longer in hospital. The average length of stay in hospital in the general population was 9.1 days/admission and in our sample 12.03 days/readmission. The bed occupancy rate for that particular year was 1.5 bed-days per person in the general population and 4.9 days per person at risk in our sample, or 3.26 times higher for spinal patients than for the general population (Table 9).

Table 9 Annual admission and bed-occupancy rates in patients with chronic SCI compared with the general population in the $\mathrm{UK}^{14}$

\begin{tabular}{lcc}
\hline & $\begin{array}{c}\text { General } \\
\text { population }\end{array}$ & $\begin{array}{c}\text { Chronic SCI } \\
\text { patients }\end{array}$ \\
\hline $\begin{array}{l}\text { Admission rate } \\
\text { (admissions per person }\end{array}$ & 0.1655 & 0.4 \\
$\quad \begin{array}{l}\text { at risk per year) } \\
\text { Bed occupancy rate } \\
\text { (bed-days per person }\end{array}$ & 1.5 & 4.9 \\
at risk per year) & & \\
\hline
\end{tabular}

Higher readmission and bed occupancy rates in the SCI population are due to increased morbidity directly related to their spinal cord injury. Therefore effective treatment results from the concentration of expertise in centres dealing exclusively with spinal cord injured patients. Admissions of such patients to general hospitals, where SCI care is not fully catered for, are frequently accompanied by common SCI related complications. Admission to specialised spinal injuries centres would minimise those unnecessary hospital acquired complications.

This study indicates that in the UK only a little over half of all readmissions is to specialised spinal injuries centres. To minimise the readmissions of patients with chronic SCI into non-specialised centres, more spinal injuries care beds would have to be available.

Furthermore, with improving survival following SCI, the prevalence of spinal cord injury is increasing, resulting in an ageing SCI population which has not existed before.

Based on the US findings that readmission incidence and bed occupancy rates decrease markedly in the first 5 years following injury and decline less rapidly or remain unchanged thereafter, it would seem reasonably safe to use the results of this study to plan future bed requirements for the population of SCI persons injured more than 5 years ago. We further believe that such a generalisation can be made as our sample was population based and thus representative.

This study only investigated 'late' readmissions. To get a complete picture of future bed requirements for all readmissions, in addition to the results reported 
here, it would be necessary to look at 'early' readmissions during the first 5 years post injury. As it has been reported by other authors that readmission rates are highest in the first years post injury, ${ }^{1,2}$ overall bed requirements for all readmissions would probably be even higher than suggested in this paper.

Another factor for which we have little data relates to readmission rates and bed occupancy during the last few years of each spinal cord injured patient's life. The original study methodology allowed the inclusion only of the patients who were still alive in 1996 followup.

Since first seen for Craig Ageing Study in 1990, 30 patients from the original sample had died by 1996 and those are the patients whose results were not available for this study. If readmission and bed occupancy rates prior to death reflect those of the general population, the overall bed requirements would be correspondingly greater.

\section{Medical reasons for hospital readmissions}

Even though the main purpose of this study was to serve as a basis for estimating future bed requirements, several other interesting points were noted.

The reasons for late hospital readmissions reflected the major causes of morbidity previously reported in the Ageing with Spinal Cord Injury Study. ${ }^{6-8}$ Urinary and skin complications, followed by digestive, musculoskeletal and nervous system complications, were the leading causes of morbidity and the main reasons for hospital readmissions in patients with chronic SCI.

Contrary to what might have been expected, the level of injury did not seem to affect either the number of readmissions or the length of stay in hospital, as no significant difference was found between paraplegic and tetraplegic patients in the rehospitalised group. It must be noted, however, that there were no ventilator dependent patients in this sample and that only four patients had C4 level of injury. The only difference by neurological grouping was that patients with very incomplete injuries (Frankel/ASIA grade D) had significantly shorter lengths of stay in hospital.

When the readmitted patients were compared with those who had not been hospitalised during the observed time period, neither the level nor the completeness of injury were significantly different between the groups. In contrast, the two groups differed in time since injury and in severity of their disability and handicap, rehospitalised patients having longer duration of paralysis, lower FIM and lower CHART scores than the non-hospitalised group.

\section{Conclusions}

A person with chronic SCI is more than twice as likely to be rehospitalised as a member of the general population in the UK and stays in hospital three times as long.
Urinary and skin complications remain the two main reasons for hospital readmissions in patients with chronic SCI, urinary complications being the most frequent and skin problems requiring the longest stay in hospital.

Risk of readmission increases with time since injury and with disability and handicap severity.

Hospital bed requirements for people with chronic spinal cord injury are greater than the amount of clinical provision currently available in specialised spinal injuries centres.

In order to meet the needs of the growing SCI population, more specialised spinal injuries care beds will be needed.

\section{Acknowledgements}

This study was supported by the Centers for Disease Control and Prevention-Disabilities Prevention Program, US, Award No R04/CCR808517-03-01, and the National Institute on Disability \& Rehabilitation Research, US, Grant No 84-133B30040. The authors would like to thank the staff of the National Spinal Injuries Centre, Stoke Mandeville, UK and the Regional Spinal Injuries Centre, Southport, UK for their help during the data collection. Our special thanks go to the patient volunteers, without whose continuous participation this study could not have been possible.

\section{References}

1 Ivie III CS, DeVivo MJ. Predicting unplanned hospitalization in persons with spinal cord injury. Arch Phys Med Rehabil 1994; 75: $1182-1188$.

2 Samsa GP, Landsman PB, Hamilton B. Inpatient hospital utilization among veterans with traumatic spinal cord injury. Arch Phys Med Rehabil 1996; 77: 1037-1043.

3 Davidoff $\mathrm{G}$ et al. Rehospitalization after initial rehabilitation for acute spinal cord injury: incidence and risk factors. Arch Phys Med Rehabil 1990; 71: $121-124$.

4 Meyers AR et al. Rehospitalization in spinal cord injury: crosssectional survey of adults living independently. Arch Phys Med Rehabil 1985; 66: $704-708$.

5 Meyers AR et al. Predictors of medical care utilization by independently living adults with spinal cord injuries. Arch Phys Med Rehabil 1989; 70: 471 - 476.

6 Whiteneck GG et al. Mortality, morbidity and psychosocial outcomes of persons spinal cord injured more than 20 years ago. Paraplegia 1992; 30: 612-630.

7 Whiteneck GG et al. (eds). Aging with Spinal Cord Injury. Demos Publications: New York, NY 1993.

8 Charlifue S, Whiteneck G, Weitzenkamp D. Secondary complications and disabilities in long-term spinal cord injury: a longitudinal follow-up of health and functional status. Final Report. Award Number RO4/CCR808517-03-01. Craig Hospital: Englewood, Colorado 1997.

9 The International Classification of Diseases. 9th Ed. Clinical Modification. Commission on Professional and Hospital Activities: Ann Arbor, Michigan 1978.

10 Frankel HL et al. The value of postural reduction in the initial management of closed injuries of the spine with paraplegia and tetraplegia. Paraplegia 1969; 7: 179-192. 
11 Maynard Jr FM et al. International standards for neurological and functional classification of spinal cord injury. Spinal Cord 1997; 35: 266-274.

12 Uniform Data System for Medical Rehabilitation. Guide for the uniform data set for medical rehabilitation (Adult FIM). State University of New York: Buffalo 1993.
13 Whiteneck GG et al. Quantifying handicap: a new measure of long-term rehabilitation outcomes. Arch Phys Med Rehabil 1992; 73: $519-526$.

14 Department of Health. Health and Personal Social Services Statistics for England. 1996 edition. The Stationery Office: London 1996. 